TRANSACTIONS OF THE

AMERICAN MATHEMATICAL SOCIETY

Volume 364, Number 12, December 2012, Pages 6459-6478

S 0002-9947(2012)05482-1

Article electronically published on June 28, 2012

\title{
A REALIZATION THEOREM FOR MODULES OF CONSTANT JORDAN TYPE AND VECTOR BUNDLES
}

\author{
DAVE BENSON AND JULIA PEVTSOVA
}

\begin{abstract}
Let $E$ be an elementary abelian $p$-group of rank $r$ and let $k$ be a field of characteristic $p$. We introduce functors $\mathcal{F}_{i}$ from finitely generated $k E$-modules of constant Jordan type to vector bundles over projective space $\mathbb{P}^{r-1}$. The fibers of the functors $\mathcal{F}_{i}$ encode complete information about the Jordan type of the module.

We prove that given any vector bundle $\mathcal{F}$ of rank $s$ on $\mathbb{P}^{r-1}$, there is a $k E$-module $M$ of stable constant Jordan type $[1]^{s}$ such that $\mathcal{F}_{1}(M) \cong \mathcal{F}$ if $p=2$, and such that $\mathcal{F}_{1}(M) \cong F^{*}(\mathcal{F})$ if $p$ is odd. Here, $F: \mathbb{P}^{r-1} \rightarrow \mathbb{P}^{r-1}$ is the Frobenius map. We prove that the theorem cannot be improved if $p$ is odd, because if $M$ is any module of stable constant Jordan type [1] $]^{s}$, then the Chern numbers $c_{1}, \ldots, c_{p-2}$ of $\mathcal{F}_{1}(M)$ are divisible by $p$.
\end{abstract}

\section{INTRODUCTION}

The class of modules of constant Jordan type was introduced by Carlson, Friedlander and the second author [5, and then consequently studied in [1, 2, 4, 6, 7, 8, The connection between modules of constant Jordan type and algebraic vector bundles on projective varieties was first observed and developed by Friedlander and the second author in [7] in the general setting of an arbitrary infinitesimal group scheme. In the present paper, we study this connection for an elementary abelian $p$-group.

Let $k$ be a field of characteristic $p$ and let $E$ be an elementary abelian $p$-group of rank $r$. We define functors $\mathcal{F}_{i}(1 \leq i \leq p)$ from finitely generated $k E$-modules of constant Jordan type to vector bundles on projective space $\mathbb{P}^{r-1}$, capturing the sum of the socles of the length $i$ Jordan blocks. The following is the main theorem of this paper.

Theorem 1.1. Given any vector bundle $\mathcal{F}$ of rank $s$ on $\mathbb{P}^{r-1}$, there exists a finitely generated $k E$-module $M$ of stable constant Jordan type $[1]^{s}$ such that

(i) if $p=2$, then $\mathcal{F}_{1}(M) \cong \mathcal{F}$;

(ii) if $p$ is odd, then $\mathcal{F}_{1}(M) \cong F^{*}(\mathcal{F})$, the pullback of $\mathcal{F}$ along the Frobenius morphism $F: \mathbb{P}^{r-1} \rightarrow \mathbb{P}^{r-1}$.

The $k E$-modules produced this way are usually large. For example, in [1, the first author showed how to produce a finitely generated $k E$-module $M$ of constant Jordan type such that $\mathcal{F}_{2}(M)$ is isomorphic to the rank two Horrocks-Mumford bundle on $\mathbb{P}^{4}$. In this case, the construction used to prove our main theorem produces a module $M$ of dimension many hundred times $p^{5}$ plus two such that

Received by the editors July 28, 2010 and, in revised form, September 28, 2010 and March 4, 2011.

2010 Mathematics Subject Classification. Primary 20C20, 14F05.

The second author was partially supported by the NSF award DMS-0800940 and DMS-0953011. 
$\mathcal{F}_{1}(M) \cong F^{*}\left(\mathcal{F}_{\mathrm{HM}}\right)$, whereas the construction in [1] produces a module of dimension $30 p^{5}$ of stable constant Jordan type $[p-1]^{30}[2]^{2}[1]^{26}$ such that applying $\mathcal{F}_{2}$ gives $\mathcal{F}_{\text {HM }}(-2)$.

The theorem for $p=2$ may be thought of as a version of the Bernstein-GelfandGelfand correspondence [3], since the group algebra of an elementary abelian 2group in characteristic two is isomorphic to an exterior algebra. But for $p$ odd it says something new and interesting. In particular, it is striking that the $p$ odd case of Theorem 1.1 cannot be strengthened to say that $\mathcal{F}_{1}(M) \cong \mathcal{F}$. The following theorem, which is proved in Section [6, gives limitations on the vector bundles appearing as $\mathcal{F}_{1}(M)$ with $M$ of stable constant Jordan type [1] $]^{s}$.

Theorem 1.2. Suppose that $M$ has stable constant Jordan type $[1]^{s}$. Then $p$ divides the Chern numbers $c_{m}\left(\mathcal{F}_{1}(M)\right)$ for $1 \leq m \leq p-2$.

The paper is organized as follows. In Section 2 we give basic definitions of the functors $\mathcal{F}_{i}$ and show that, applied to modules of constant Jordan type, they produce algebraic vector bundles. Section 3 , together with Lemmas 5.1 and 5.2, analyze behavior of the functors $\mathcal{F}_{i}$ with respect to Heller shifts and duals. This analysis plays a key role in the proof of our main theorem. In Section 4 we construct a functor from the bounded derived category of coherent $\mathcal{O}_{\mathbb{P}^{r-1}}$-modules to the stable module category of $k E$. This functor is employed in the proof of Theorem 1.1. which occupies Section 5. Finally, in Section 6 we discuss Chern classes of the functors $\mathcal{F}_{i}$ and prove Theorem 1.2 .

\section{Definition of the FunCtors $\mathcal{F}_{i}$}

Let $k$ be a perfect field of characteristic $p$. Let $E=\left\langle g_{1}, \ldots, g_{r}\right\rangle$ be an elementary abelian $p$-group of rank $r$, and set $X_{i}=g_{i}-1 \in k E$ for $1 \leq i \leq r$. Let $J(k E)=$ $\left\langle X_{1}, \ldots, X_{r}\right\rangle$ be the augmentation ideal of $k E$. The images of $X_{1}, \ldots, X_{r}$ form a basis for $J(k E) / J^{2}(k E)$, which we think of as an affine space $\mathbb{A}_{k}^{r}$ over $k$. Let $K / k$ be a field extension. If $0 \neq \alpha=\left(\lambda_{1}, \ldots, \lambda_{r}\right) \in \mathbb{A}_{K}^{r}$, we define

$$
X_{\alpha}=\lambda_{1} X_{1}+\cdots+\lambda_{r} X_{r} \in K E .
$$

This is an element of $J(K E)$ satisfying $X_{\alpha}^{p}=0$. If $M$ is a finitely generated $k E$ module, then $X_{\alpha}$ acts nilpotently on $M_{K}=M \otimes K$, and we can decompose $M_{K}$ into Jordan blocks. They all have eigenvalue zero, and length between 1 and $p$. We say that $M$ has constant Jordan type $[p]^{a_{p}} \ldots[1]^{a_{1}}$ if there are $a_{p}$ Jordan blocks of length $p, \ldots, a_{1}$ blocks of length 1 , independently of the choice of $\alpha$. Since $a_{p}$ is determined by $a_{p-1}, \ldots, a_{1}$ and the dimension of $M$, we also say that $M$ has stable constant Jordan type $[p-1]^{a_{p-1}} \ldots[1]^{a_{1}}$. Note that the property of having constant Jordan type and the type itself do not depend on the choice of generators $\left\langle g_{1}, \ldots, g_{r}\right\rangle$ (see [5]).

We write $k\left[Y_{1}, \ldots, Y_{r}\right]$ for the coordinate ring $k\left[\mathbb{A}^{r}\right]$, where the $Y_{i}$ are the linear functions defined by $Y_{i}\left(X_{j}\right)=\delta_{i j}$ (Kronecker delta). We write $\mathbb{P}^{r-1}$ for the corresponding projective space. Let $\mathcal{O}$ be the structure sheaf on $\mathbb{P}^{r-1}$. If $\mathcal{F}$ is a sheaf of $\mathcal{O}$-modules and $j \in \mathbb{Z}$, we write $\mathcal{F}(j)$ for the $j$ th Serre twist $\mathcal{F} \otimes_{\mathcal{O}} \mathcal{O}(j)$. If $M$ is a finitely generated $k E$-module, we write $\widetilde{M}$ for the trivial vector bundle $M \otimes_{k} \mathcal{O}$, so that $\widetilde{M}(j)=M \otimes_{k} \mathcal{O}(j)$. Friedlander and the second author [7, $\S 4$ ] define a map of 
vector bundles $\theta_{M}: \widetilde{M} \rightarrow \widetilde{M}(1)$ by the formula

$$
\theta_{M}(m \otimes f)=\sum_{i=1}^{r} X_{i}(m) \otimes Y_{i} f .
$$

By abuse of notation we also write $\theta_{M}$ for the twist $\theta_{M}(j): \widetilde{M}(j) \rightarrow \widetilde{M}(j+1)$. With this convention we have $\theta_{M}^{p}=0$.

We define functors $\mathcal{F}_{i, j}$ for $0 \leq j<i \leq p$ from finitely generated $k E$-modules to coherent sheaves on $\mathbb{P}^{r-1}$ by taking the following subquotients of $\widetilde{M}$ :

$$
\mathcal{F}_{i, j}(M)=\frac{\operatorname{Ker} \theta_{M}^{j+1} \cap \operatorname{Im} \theta_{M}^{i-j-1}}{\left(\operatorname{Ker} \theta_{M}^{j+1} \cap \operatorname{Im} \theta_{M}^{i-j}\right)+\left(\operatorname{Ker} \theta_{M}^{j} \cap \operatorname{Im} \theta_{M}^{i-j-1}\right)} .
$$

We then define

$$
\mathcal{F}_{i}(M)=\mathcal{F}_{i, 0}(M)=\frac{\operatorname{Ker} \theta_{M} \cap \operatorname{Im} \theta_{M}^{i-1}}{\operatorname{Ker} \theta_{M} \cap \operatorname{Im} \theta_{M}^{i}} .
$$

For a point $0 \neq \alpha \in \mathbb{A}^{r}$ and the corresponding operator $X_{\alpha}: M \rightarrow M$, we also define

$$
\mathcal{F}_{i, \alpha}(M)=\frac{\operatorname{Ker} X_{\alpha} \cap \operatorname{Im} X_{\alpha}^{i-1}}{\operatorname{Ker} X_{\alpha} \cap \operatorname{Im} X_{\alpha}^{i}} .
$$

Note that $\mathcal{F}_{i, \alpha}(M)$ is evidently well-defined for $\bar{\alpha} \in \mathbb{P}^{r-1}$.

In the next proposition we show that functors $\mathcal{F}_{i}$ take modules of constant Jordan type to algebraic vector bundles (equivalently, locally free sheaves), and that they commute with specialization.

\section{Proposition 2.1.}

(1) Let $M$ be a kE-module of constant Jordan type $[p]^{a_{p}} \ldots[1]^{a_{1}}$. Then the sheaf $\mathcal{F}_{i}(M)$ is locally free of rank $a_{i}$.

(2) Let $f: M \rightarrow N$ be a map of modules of constant Jordan type. For any point $\bar{\alpha}=\left[\lambda_{1}: \cdots: \lambda_{r}\right] \in \mathbb{P}^{r-1}$ with residue field $k(\bar{\alpha})$ we have a commutative diagram

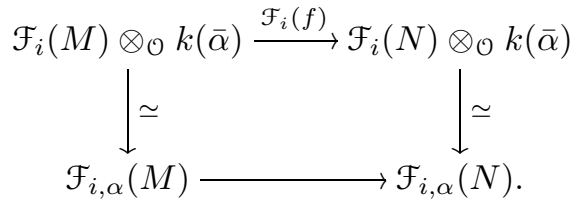

Proof. (1). Since the module $M$ is fixed throughout the proof, we shall use $\theta$ to denote $\theta_{M}$.

Note that $\operatorname{Ker} \theta \cap \operatorname{Im} \theta^{i}=\operatorname{Ker}\left\{\theta: \operatorname{Im} \theta^{i} \rightarrow \operatorname{Im} \theta^{i+1}\right\}$. Hence, we have a short exact sequence

$$
0 \longrightarrow \operatorname{Ker} \theta \cap \operatorname{Im} \theta^{i} \longrightarrow \operatorname{Im} \theta^{i} \stackrel{\theta}{\longrightarrow} \operatorname{Im} \theta^{i+1} \longrightarrow 0 .
$$

Since $M$ has constant Jordan type, $\operatorname{Im} \theta^{i}$ is locally free by [7, 4.13]. Therefore, specialization of the sequence (2.1.1) at any point $\bar{\alpha}=\left[\lambda_{1}: \cdots: \lambda_{r}\right]$ of $\mathbb{P}^{r-1}$ yields a short exact sequence of vector spaces

$$
0 \rightarrow\left(\operatorname{Ker} \theta \cap \operatorname{Im} \theta^{i}\right) \otimes_{\mathcal{O}} k(\bar{\alpha}) \rightarrow \operatorname{Im} \theta^{i} \otimes_{\mathcal{O}} k(\bar{\alpha}) \rightarrow \operatorname{Im} \theta^{i+1} \otimes_{\mathcal{O}} k(\bar{\alpha}) \rightarrow 0 .
$$


By [7, 4.13], $\operatorname{Im} \theta^{i} \otimes_{\mathcal{O}} k(\bar{\alpha}) \simeq \operatorname{Im}\left\{X_{\alpha}^{i}: M \rightarrow M\right\}$. In particular, the dimension of fibers of $\operatorname{Im} \theta^{i}$ is constant and equals $\sum_{j=i+1}^{p} a_{j}(j-i)$. We can rewrite the sequence (2.1.2) as

$$
0 \longrightarrow\left(\operatorname{Ker} \theta \cap \operatorname{Im} \theta^{i}\right) \otimes_{\mathcal{O}} k(\bar{\alpha}) \longrightarrow \operatorname{Im} X_{\alpha}^{i} \stackrel{X_{\alpha}}{\longrightarrow} \operatorname{Im} X_{\alpha}^{i+1} \longrightarrow 0 .
$$

Hence, the fiber of $\operatorname{Ker} \theta \cap \operatorname{Im} \theta^{i}$ at a point $\bar{\alpha}$ equals $\operatorname{Ker} X_{\alpha} \cap \operatorname{Im} X_{\alpha}^{i}$. In particular, $\operatorname{Ker} \theta \cap \operatorname{Im} \theta^{i}$ has fibers of constant dimension equal to

$$
\sum_{j=i+1}^{p} a_{j}(j-i)-\sum_{j=i+2}^{p} a_{j}(j-i-1)=\sum_{j=i+1}^{p} a_{j} .
$$

Applying [7, 4.11] (see also [10, V. ex. 5.8]), we conclude that $\operatorname{Ker} \theta \cap \operatorname{Im} \theta^{i}$ is locally free of rank $\sum_{j=i+1}^{p} a_{j}$.

Consider the short exact sequence that defines $\mathcal{F}_{i}(M)$ :

$$
0 \longrightarrow \operatorname{Ker} \theta \cap \operatorname{Im} \theta^{i} \longrightarrow \operatorname{Ker} \theta \cap \operatorname{Im} \theta^{i-1} \longrightarrow \mathcal{F}_{i}(M) \longrightarrow 0 .
$$

Specializing at $\bar{\alpha}$, we get

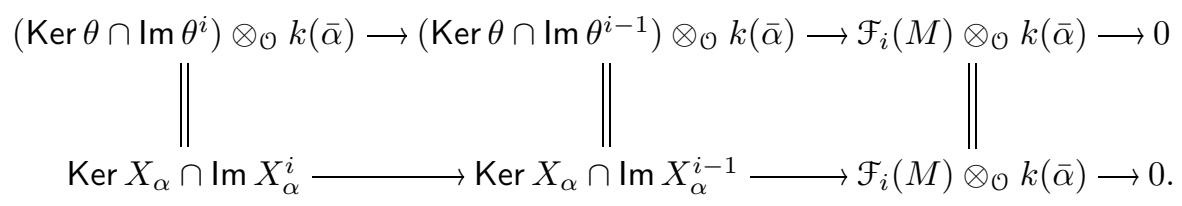

The first arrow of the bottom row is clearly an injection. Hence,

$$
\operatorname{dim}\left(\mathcal{F}_{i}(M) \otimes_{\mathcal{O}} k(\bar{\alpha})\right)=\sum_{j=i}^{p} a_{j}-\sum_{j=i+1}^{p} a_{j}=a_{i}
$$

for any point $\alpha \in \mathbb{P}^{r-1}$. Applying [7, 4.11] again, we conclude that $\mathcal{F}_{i}(M)$ is locally free (of rank $a_{i}$ ).

Statement (2) follows immediately by applying the last diagram to both $M$ and $N$.

Lemma 2.2. $\widetilde{M}$ has a filtration in which the filtered quotients are isomorphic to $\mathcal{F}_{i, j}(M)$ for $0 \leq j<i \leq p$.

Proof. We consider two filtrations on $\widetilde{M}$, the "kernel filtration" and the "image filtration":

$$
\begin{gathered}
0 \subset \operatorname{Ker} \theta_{M} \subset \ldots \subset \operatorname{Ker} \theta_{M}^{p-1} \subset \widetilde{M} \\
0=\operatorname{Im} \theta_{M}^{p} \subset \operatorname{Im} \theta_{M}^{p-1} \subset \ldots \subset \operatorname{Im} \theta_{M} \subset \operatorname{Im} \theta_{M}^{0}=\widetilde{M} .
\end{gathered}
$$

To simplify notation, we set $\mathcal{K}_{j}=\operatorname{Ker} \theta_{M}^{i}$ and $\mathcal{J}_{i}=\operatorname{Im} \theta_{M}^{p-i}$. Using the standard refinement procedure, we refine the kernel filtration by the image filtration:

$\mathcal{K}_{j} \subset\left(\mathcal{K}_{j+1} \cap \mathcal{J}_{1}\right)+\mathcal{K}_{j} \subset \ldots \subset\left(\mathcal{K}_{j+1} \cap \mathcal{J}_{\ell}\right)+\mathcal{K}_{j} \subset\left(\mathcal{K}_{j+1} \cap \mathcal{J}_{\ell+1}\right)+\mathcal{K}_{j} \subset \ldots \subset \mathcal{K}_{j+1}$. 
For any three sheaves $A, B, C$ with $B \subset A$, the second isomorphism theorem and the modular law imply that

$$
\frac{A+C}{B+C} \simeq \frac{A+(B+C)}{B+C} \simeq \frac{A}{A \cap(B+C)} \simeq \frac{A}{B+(A \cap C)} .
$$

Hence, we can identify the subquotients of the refined kernel filtration above as

$$
\frac{\left(\mathcal{K}_{j+1} \cap \mathcal{J}_{\ell+1}\right)+\mathcal{K}_{j}}{\left(\mathcal{K}_{j+1} \cap \mathcal{J}_{\ell}\right)+\mathcal{K}_{j}} \simeq \frac{\mathcal{K}_{j+1} \cap \mathcal{J}_{\ell+1}}{\left(\mathcal{K}_{j+1} \cap \mathcal{J}_{\ell}\right)+\left(\mathcal{K}_{j} \cap \mathcal{J}_{\ell+1}\right)} .
$$

Setting $i=p-\ell+j$, we get that the latter quotient is precisely $\mathcal{F}_{i, j}(M)$ (note that when $j>\ell$, the corresponding subquotient is trivial).

Lemma 2.3. For $0 \leq j<i$, we have a natural isomorphism $\mathcal{F}_{i, j}(M) \cong \mathcal{F}_{i}(M)(j)$.

Proof. For $0<j<i$, the map $\theta_{M}: \widetilde{M} \rightarrow \widetilde{M}(1)$ induces a natural isomorphism $\mathcal{F}_{i, j}(M) \rightarrow \mathcal{F}_{i, j-1}(M)(1)$. Since $\mathcal{F}_{i, 0}=\mathcal{F}_{i}$, the result follows by induction on $j$.

Remark 2.4. It follows from the proof of Proposition 2.1 that the subquotient functors $\mathcal{F}_{i, j}$ are linked as follows:

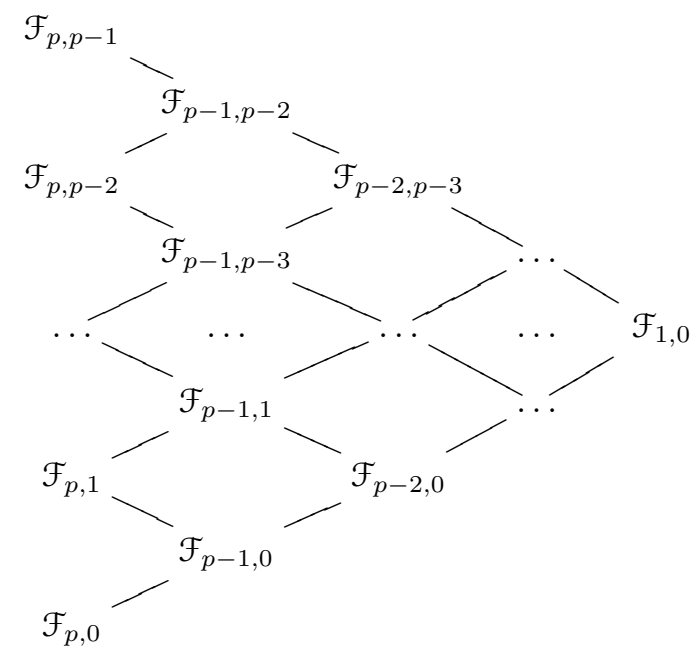

We finish this section with an example.

Example 2.5. Let $M=k E / J^{2}(k E)$. Then $M$ has constant Jordan type [2][1] ${ }^{r-1}$. In the short exact sequence of vector bundles

$$
0 \rightarrow \widetilde{M / \operatorname{Rad} M} \stackrel{\theta}{\rightarrow} \widetilde{\operatorname{Rad} M}(1) \rightarrow \mathcal{F}_{1}(M)(1) \rightarrow 0
$$

the map $\theta$ (induced by $\theta_{M}$ ) is equal to the map defining the tangent bundle (or sheaf of derivations) $\mathcal{T}$ of $\mathbb{P}^{r-1}$ :

$$
0 \rightarrow \mathcal{O} \rightarrow \mathcal{O}(1)^{r} \rightarrow \mathcal{T} \rightarrow 0 .
$$

It follows that $\mathcal{F}_{1}(M) \cong \mathcal{T}(-1)$. On the other hand we have $\mathcal{F}_{2,1}(M) \cong \mathcal{O}$, and hence $\mathcal{F}_{2}(M) \cong \mathcal{O}(-1)$. 


\section{TWISTS AND SYZYGIES}

We need a general lemma whose proof we provide for completeness.

Lemma 3.1. Let $X$ be a Noetherian scheme over $k$, and let $M, N$ be locally free $\mathcal{O}_{X}$-modules. Let $f: M \rightarrow N$ be a morphism of $\mathcal{O}_{X}$-modules such that

$$
f \otimes_{\mathcal{O}_{X}} k(x): M \otimes_{\mathcal{O}_{X}} k(x) \rightarrow N \otimes_{\mathcal{O}_{X}} k(x)
$$

is an isomorphism for any $x \in X$. Then $f$ is an isomorphism.

Proof. It suffices to show that $f$ induces an isomorphism on stalks. Hence, we may assume that $X=\operatorname{Spec} R$, where $R$ is a local ring with the maximal ideal $\mathfrak{m}$, and $M, N$ are free modules. Since specialization is right exact, $f$ is surjective by Nakayama's lemma. Hence, we have an exact sequence of $R$-modules:

$$
0 \rightarrow \operatorname{ker} f \rightarrow M \rightarrow N \rightarrow 0 .
$$

Since $N$ is free, $\operatorname{Tor}_{1}^{R}(N, R / \mathfrak{m})$ vanishes, and hence $\operatorname{ker} f \otimes_{R} R / \mathfrak{m}=0$. By Nakayama's lemma, $\operatorname{ker} f=0$; therefore, $f$ is injective.

Theorem 3.2. Let $M$ be a finite-dimensional $k E$-module and let $1 \leq i \leq p-1$. Then there is a natural isomorphism

$$
\mathcal{F}_{i}(M)(-p+i) \cong \mathcal{F}_{p-i}(\Omega M) .
$$

Proof. Consider the diagram

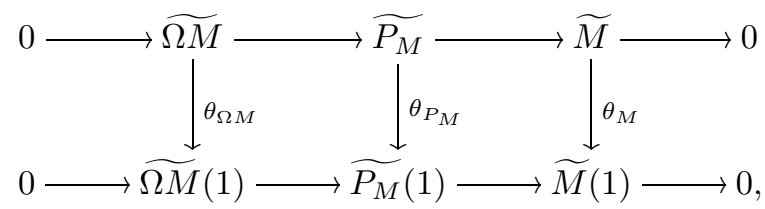

where $P_{M}$ is a projective cover of $M$. Let

$$
\delta: \operatorname{Ker} \theta_{M} \rightarrow \operatorname{Coker} \theta_{\Omega M}
$$

be the switchback map. A simple diagram chase in conjunction with the fact that $\theta_{P_{M}}^{p}=0$ yields that the restriction of $\delta$ to $\operatorname{Ker} \theta_{M} \cap \operatorname{Im} \theta_{M}^{i-1}$ lands in

$$
\frac{\operatorname{Ker} \theta_{\Omega M}^{p-i}}{\operatorname{Ker} \theta_{\Omega M}^{p-i} \cap \operatorname{Im} \theta_{\Omega(M)}}(1) \text {. }
$$

Projecting the latter onto

$$
\mathcal{F}_{p-i, p-i-1}(\Omega M)(1)=\frac{\operatorname{Ker} \theta_{\Omega M}^{p-i}}{\operatorname{Ker} \theta_{\Omega M}^{p-i-1}+\operatorname{Ker} \theta_{\Omega M}^{p-i} \cap \operatorname{Im} \theta_{\Omega(M)}}(1),
$$

we get a map of bundles:

$$
\delta^{\prime}: \operatorname{Ker} \theta_{M} \cap \operatorname{Im} \theta_{M}^{i-1} \rightarrow \mathcal{F}_{p-i, p-i-1}(\Omega M)(1) .
$$

Since $\delta^{\prime}$ evidently kills $\operatorname{Ker} \theta_{M} \cap \operatorname{Im} \theta_{M}^{i}$, we conclude that $\delta^{\prime}$ factors through $\mathcal{F}_{i}(M)$. Hence, we have an induced map

$$
\bar{\delta}: \mathcal{F}_{i}(M) \rightarrow \mathcal{F}_{p-i, p-i-1}(\Omega M)(1) .
$$


A simple block count shows that this is an isomorphism at each fiber. Hence, by Lemma 3.1, this is an isomorphism of bundles. Thus using Lemma 2.3 (i.e., applying $\theta_{\Omega M}$ a further $p-i-1$ times), we have

$$
\mathcal{F}_{i}(M) \cong \mathcal{F}_{p-i, p-i-1}(\Omega M)(1) \cong \mathcal{F}_{p-i}(\Omega M)(p-i) .
$$

Twisting by $\mathcal{O}(-p+i)$, we get the desired isomorphism.

Let $f: M \rightarrow N$ be a map of $k E$-modules. The naturality of the isomorphism

$$
\mathcal{F}_{i}(M)(-p+i) \cong \mathcal{F}_{p-i}(\Omega M)
$$

is equivalent to the commutativity of the diagram

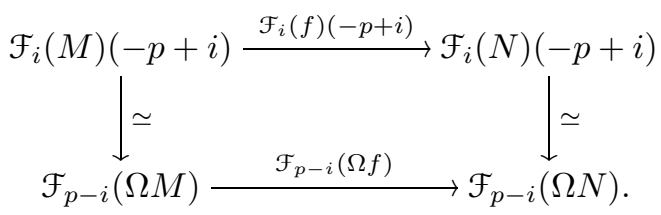

The commutativity follows from the construction of the map $\bar{\delta}$ and naturality of the "shifting isomorphism" of Lemma 2.3 .

Corollary 3.3. Let $M$ be a finite-dimensional $k E$-module and let $1 \leq i \leq p-1$. Then $\mathcal{F}_{i}\left(\Omega^{2} M\right) \cong \mathcal{F}_{i}(M)(-p)$.

Proof. Apply the theorem twice.

Corollary 3.4. We have $\mathcal{F}_{1}\left(\Omega^{2 n} k\right) \cong \mathcal{O}(-n p)$, and $\mathcal{F}_{p-1}\left(\Omega^{2 n-1} k\right) \cong \mathcal{O}(1-n p)$.

Proof. This follows from the theorem and the corollary, using the isomorphism $\mathcal{F}_{1}(k) \cong \mathcal{O}$.

Remark 3.5. If $p=2$, then Theorem 3.2 and Corollary 3.4 reduce to the statements that $\mathcal{F}_{1}(\Omega M) \cong \mathcal{F}_{1}(M)(-1)$ and $\mathcal{F}_{1}\left(\Omega^{n} k\right) \cong \mathcal{O}(-n)$.

For a coherent sheaf $\mathcal{E}$, we denote by $\mathcal{E}^{\vee}=\mathcal{H}_{o} m_{\mathcal{O}}(\mathcal{E}, \mathcal{O})$ the dual sheaf.

Theorem 3.6. Let $M^{*}$ be the $k$-linear dual of $M$, as a $k E$-module. Then

$$
\mathcal{F}_{i}\left(M^{*}\right) \cong \mathcal{F}_{i}(M)^{\vee}(-i+1) .
$$

Proof. We shall first establish the following isomorphism:

$$
\mathcal{F}_{i, i-1}\left(M^{*}\right) \cong \mathcal{F}_{i, 0}(M)^{\vee}
$$

By definition, $\mathcal{F}_{i, i-1}\left(M^{*}\right) \simeq \frac{\operatorname{Ker} \theta_{M^{*}}^{i}}{\operatorname{Ker} \theta_{M^{*}}^{i} \cap \operatorname{lm} \theta_{M^{*}}+\operatorname{Ker} \theta_{M^{*}}^{i-1}}$. Let $\phi \in \operatorname{Ker} \theta_{M^{*}}^{i}$. Hence $\phi$ : $\widetilde{M} \rightarrow \mathcal{O}$ is an $\mathcal{O}$-linear map such that $\phi \circ \theta_{M}^{i}=0$. Restricting $\phi$ to $\operatorname{Ker} \theta_{M} \cap \operatorname{Im} \theta_{M}^{i-1}$ we obtain a linear map

$$
\phi^{\prime}: \operatorname{Ker} \theta_{M} \cap \operatorname{Im} \theta_{M}^{i-1} \rightarrow \mathcal{O} .
$$

The condition $\phi \circ \theta_{M}^{i}=0$ immediately implies that $\phi^{\prime}$ vanishes on $\operatorname{Ker} \theta_{M} \cap \operatorname{Im} \theta_{M}^{i}$. Hence, it induces a linear map on the quotient:

$$
\bar{\phi}: \mathcal{F}_{i}(M)=\frac{\operatorname{Ker} \theta_{M} \cap \operatorname{lm} \theta_{M}^{i-1}}{\operatorname{Ker} \theta_{M} \cap \operatorname{lm} \theta_{M}^{i}} \longrightarrow \mathcal{O} .
$$


We define

$$
\Phi: \operatorname{Ker} \theta_{M^{*}}^{i} \longrightarrow \operatorname{Hom}_{\mathcal{O}}\left(\mathcal{F}_{i}(M), \mathcal{O}\right)=\mathcal{F}_{i}(M)^{\vee}
$$

via

$$
\phi \mapsto \bar{\phi}
$$

Suppose that $\phi \in \operatorname{Ker} \theta_{M^{*}}^{i-1}$. Then $\phi \circ \theta_{M}^{i-1}=0$ and, hence, $\phi^{\prime}=0$. Now suppose that $\phi \in \operatorname{Ker} \theta_{M^{*}}^{i} \cap \operatorname{Im} \theta_{M^{*}}$. Then $\phi=\psi \circ \theta_{M}$ for some linear map $\psi: \widetilde{M} \rightarrow \mathcal{O}$. This implies that $\phi \downarrow_{\operatorname{Ker} \theta_{M}}=0$. Therefore, $\phi^{\prime}=0$. We have thus established that $\Phi$ vanishes on $\operatorname{Ker} \theta_{M^{*}}^{i-1}+\operatorname{Ker} \theta_{M^{*}}^{i} \cap \operatorname{Im} \theta_{M^{*}}$. Hence, it induces a map

$$
\bar{\Phi}: \mathcal{F}_{i, i-1}\left(M^{*}\right)=\frac{\operatorname{Ker} \theta_{M^{*}}^{i}}{\operatorname{Ker} \theta_{M^{*}}^{i-1}+\operatorname{Ker} \theta_{M^{*}}^{i} \cap \operatorname{lm} \theta_{M^{*}}} \longrightarrow \mathcal{F}_{i}(M)^{\vee} .
$$

It follows from construction that the map is $\mathcal{O}$-linear. A simple block count shows that it induces an isomorphism on fibers. Hence, $\bar{\Phi}$ is an isomorphism.

The theorem now follows from (3.6.1) together with Lemma 2.3.

We finish this section with exactness properties of the functors $\mathcal{F}_{i}$, which will be essential in the proof of the main theorem.

Let $\mathcal{C}(k E)$ be the exact category of modules of constant Jordan type as introduced in [4. This is an exact category in the sense of Quillen: the objects are finitedimensional $k E$-modules of constant Jordan type, and the admissible morphisms are morphisms which can be completed to a locally split short exact sequence. We call a sequence of $k E$-modules

$$
0 \longrightarrow M_{1} \longrightarrow M_{2} \longrightarrow M_{3} \longrightarrow 0
$$

locally split if it is split upon restriction to $k\left[X_{\alpha}\right] / X_{\alpha}^{p}$ for any $0 \neq \alpha \in \mathbb{A}^{r}$.

Proposition 3.7. The functor $\mathcal{F}_{i}: \mathcal{C}(k E) \rightarrow \operatorname{Coh}\left(\mathbb{P}_{k}^{r-1}\right)$ is exact for $1 \leq i \leq p-1$.

Proof. Let

$$
0 \longrightarrow M_{1} \longrightarrow M_{2} \longrightarrow M_{3} \longrightarrow 0
$$

be a locally split short exact sequence of modules of constant Jordan type. Then the Jordan type of the middle term is the sum of Jordan types of the end terms. Hence, $\operatorname{rk} \mathcal{F}_{i}\left(M_{2}\right)=\operatorname{rk} \mathcal{F}_{i}\left(M_{1}\right)+\operatorname{rk} \mathcal{F}_{i}\left(M_{3}\right)$ for any $i$. Consider the map $\mathcal{F}_{i}\left(M_{2}\right) \rightarrow$ $\mathcal{F}_{i}\left(M_{3}\right)$. By Proposition 2.1, the specialization $\mathcal{F}_{i}\left(M_{2}\right) \otimes_{\mathcal{O}} k(\bar{\alpha}) \rightarrow \mathcal{F}_{i}\left(M_{3}\right) \otimes_{\mathcal{O}} k(\bar{\alpha})$ is surjective at any point $\bar{\alpha} \in \mathbb{P}^{r-1}$. Arguing as in Lemma 3.1, we conclude that $\mathcal{F}_{i}\left(M_{2}\right) \rightarrow \mathcal{F}_{i}\left(M_{3}\right)$ is surjective. Similarly, we show that $\mathcal{F}_{i}\left(M_{1}\right) \rightarrow \mathcal{F}_{i}\left(M_{2}\right)$ is injective. Finally, the equality $\operatorname{rk} \mathcal{F}_{i}\left(M_{2}\right)=\operatorname{rk} \mathcal{F}_{i}\left(M_{1}\right)+\operatorname{rk} \mathcal{F}_{i}\left(M_{3}\right)$ implies exactness in the middle term.

\section{The COnstruction}

In this section we construct a functor

$$
\mathcal{G}: \mathrm{D}^{\mathrm{b}}(\mathcal{O} \text {-mod }) \rightarrow k E \text {-stmod }
$$

from the bounded derived category of coherent $\mathcal{O}$-modules to the stable module category of $k E$.

Since $H^{1}(E, k)$ is the vector space dual of $J(k E) / J^{2}(k E)$, there are elements $y_{1}, \ldots, y_{r}$ forming a vector space basis for $H^{1}(E, k)$ and corresponding to the linear functions $Y_{1}, \ldots, Y_{r}$ on $J(k E) / J^{2}(k E)$ introduced in Section 2. For $p>2$, we 
write $\beta: H^{1}(E, k) \rightarrow H^{2}(E, k)$ for the Bockstein map, and we set $x_{i}=\beta\left(y_{i}\right)$. The cohomology ring $H^{*}(E, k)$ has the following structure:

$$
H^{*}(E, k)= \begin{cases}k\left[y_{1}, \ldots, y_{r}\right] & p=2 \\ \Lambda\left(y_{1}, \ldots, y_{r}\right) \otimes k\left[x_{1}, \ldots, x_{r}\right] & p \text { odd }\end{cases}
$$

where $\operatorname{deg}\left(y_{i}\right)=1$ and $\operatorname{deg}\left(x_{i}\right)=2$.

We shall write $\mathrm{Hom}$ for the Hom in the stable module category. The isomorphism $H^{1}(E, k) \simeq \underline{\operatorname{Hom}}\left(\Omega^{n+1} k, \Omega^{n} k\right)$ implies that for a generator $y_{i} \in H^{1}(E, k)$ we have a corresponding map $y_{i}: \Omega^{n+1} k \rightarrow \Omega^{n} k$ in the stable module category, and similarly for $x_{i}$ for $p$ odd. For our construction, we need to choose canonical representatives of these maps so that they strictly commute, that is, commute as maps in $k E$-mod, not just in the stable module category. We proceed to describe such a choice.

Let $C=\langle g\rangle$ be a cyclic group of order $p$, and let $\mathbf{C}$ be the periodic projective resolution of the trivial $k C$-module $k$ which is given by

$$
\cdots k C \stackrel{1-g}{\longrightarrow} k C \stackrel{1+g+\cdots+g^{p-1}}{\longrightarrow} k C \stackrel{1-g}{\longrightarrow} k C \rightarrow 0 .
$$

If $p=2$, this is periodic of period one, and there is a degree one map $\hat{y}: \mathbf{C} \rightarrow \mathbf{C}$ representing $y \in H^{1}(C, k)$. If $p$ is odd, it has period two, and there is a degree two map $\hat{x}: \mathbf{C} \rightarrow \mathbf{C}$ representing $x \in H^{2}(C, k)$.

Now if $E=\left\langle g_{1}, \ldots, g_{r}\right\rangle \cong(\mathbb{Z} / p)^{r}$, we let

$$
\mathbf{P}=\underbrace{\mathbf{C} \otimes \cdots \otimes \mathbf{C}}_{r}
$$

Then $\mathbf{P}$ is a minimal projective resolution of $k$ as a $k E$-module. Let

$$
\begin{array}{ll}
\hat{y}_{i}=1 \otimes \cdots \otimes \hat{y} \otimes \cdots \otimes 1: \mathbf{P} \rightarrow \mathbf{P} & (p=2), \\
\hat{x}_{i}=1 \otimes \cdots \otimes \hat{x} \otimes \cdots \otimes 1: \mathbf{P} \rightarrow \mathbf{P} & (p \text { odd }) .
\end{array}
$$

Here, all terms in the tensor product are equal to one except the $i$ th term which is $\hat{y}$, respectively $\hat{x}$. The elements $\hat{y}_{i}$ and $\hat{x}_{i}$ represent the elements $y_{i} \in H^{1}(E, k)$ $(p=2)$, respectively $x_{i} \in H^{2}(E, k)$ ( $p$ odd). These maps commute by construction, and so we get a ring homomorphism

$$
\begin{aligned}
& k\left[y_{1}, \ldots, y_{r}\right] \rightarrow \operatorname{Hom}_{k E}^{*}(\mathbf{P}, \mathbf{P}) \quad(p=2), \\
& k\left[x_{1}, \ldots, x_{r}\right] \rightarrow \operatorname{Hom}_{k E}^{*}(\mathbf{P}, \mathbf{P}) \quad(p \text { odd }) .
\end{aligned}
$$

Since $\Omega^{n}(k)$ is the $n$th kernel in the complex $\mathbf{P}$, we get induced maps which by abuse of notation we write as

$$
\begin{aligned}
& y_{i}: \Omega^{n+1}(k) \rightarrow \Omega^{n}(k) \quad(p=2), \\
& x_{i}: \Omega^{n+2}(k) \rightarrow \Omega^{n}(k) \quad(p \text { odd })
\end{aligned}
$$

for all $n \geq 0$. Dualizing, we get these maps for all $n \in \mathbb{Z}$. They strictly commute, in the sense that $y_{i} y_{j}$ and $y_{j} y_{i}$ give the same map $\Omega^{n+2}(k) \rightarrow \Omega^{n}(k)(p=2)$, and $x_{i} x_{j}$ and $x_{j} x_{i}$ give the same map $\Omega^{n+4}(k) \rightarrow \Omega^{n}(k)$ ( $p$ odd).

Let $\mathbf{C}$ be a complex in $\mathrm{D}^{\mathrm{b}}(\mathrm{O}$-mod). By the Hilbert syzygy theorem, the CartanEilenberg resolution of $\mathbf{C}$ can be taken to have entries of the form $\bigoplus_{j=1}^{\ell} \mathcal{O}\left(i_{j}\right)$. Hence, we can replace $\mathbf{C}$ with a quasi-isomorphic complex which has the form

$$
\bigoplus_{j=1}^{m_{n}} \mathcal{O}\left(a_{n, j}\right) \rightarrow \cdots \rightarrow \bigoplus_{j=1}^{m_{1}} \mathcal{O}\left(a_{1, j}\right) \rightarrow \bigoplus_{j=1}^{m_{0}} \mathcal{O}\left(a_{0, j}\right) .
$$


Each of the maps in this complex is a matrix whose entries are homogeneous polynomials in $Y_{1}, \ldots, Y_{r}$. Replacing each $Y_{i}$ with $y_{i}$ if $p=2$ and with $x_{i}$ if $p$ is odd, where $y_{i}$ and $x_{i}$ are the maps defined in (4.0.1), gives matrices of homogeneous polynomials on $y_{i}$ for $p=2$ (and $x_{i}$ for $p$ odd) which we may use to form the following sequence of objects and maps in $k E$-mod:

$$
\bigoplus_{j=1}^{m_{n}} \Omega^{-\varepsilon a_{n, j}}(k) \rightarrow \cdots \rightarrow \bigoplus_{j=1}^{m_{1}} \Omega^{-\varepsilon a_{1, j}}(k) \rightarrow \bigoplus_{j=1}^{m_{0}} \Omega^{-\varepsilon a_{0, j}}(k),
$$

where $\varepsilon=1$ if $p=2$ and $\varepsilon=2$ if $p$ is odd. Since the maps $y_{i}(p=2)$, respectively $x_{i}$ ( $p$ odd), strictly commute, we get a complex in $k E$-mod and, hence, an object in $\mathrm{D}^{\mathrm{b}}(k E$-mod). We call the resulting complex $\widetilde{\mathcal{G}}(\mathbf{C})$.

Note that chain maps between bounded complexes of coherent $\mathcal{O}$-modules which are direct sums of Serre twists of the structure sheaf and a chain homotopy between such chain maps are all given by matrices of polynomials on $Y_{i}$, so that they are realized as chain maps and chain homotopies in $\mathrm{D}^{\mathrm{b}}(k E$-mod). This observation implies the following proposition, a detailed proof of which we leave as an exercise for an interested reader.

Proposition 4.1. The association

$$
\widetilde{\mathcal{G}}: \mathrm{D}^{\mathrm{b}}(\mathcal{O} \text {-mod }) \rightarrow \mathrm{D}^{\mathrm{b}}(k E-\bmod )
$$

which sends a bounded complex of coherent $\mathcal{O}$-modules quasi-isomorphic to

$$
\bigoplus_{j=1}^{m_{n}} \mathcal{O}\left(a_{n, j}\right) \rightarrow \cdots \rightarrow \bigoplus_{j=1}^{m_{1}} \mathcal{O}\left(a_{1, j}\right) \rightarrow \bigoplus_{j=1}^{m_{0}} \mathcal{O}\left(a_{0, j}\right)
$$

to the complex

$$
\bigoplus_{j=1}^{m_{n}} \Omega^{-\varepsilon a_{n, j}}(k) \rightarrow \cdots \rightarrow \bigoplus_{j=1}^{m_{1}} \Omega^{-\varepsilon a_{1, j}}(k) \rightarrow \bigoplus_{j=1}^{m_{0}} \Omega^{-\varepsilon a_{0, j}}(k)
$$

in $\mathrm{D}^{\mathrm{b}}(k E-\mathrm{mod})$ as defined in (4.0.3) is a triangulated functor.

We denote by $\mathbb{K}^{b}$ (proj) the category of perfect complexes over $k E$, that is, the thick subcategory of $\mathrm{D}^{\mathrm{b}}(k E$-mod) consisting of complexes quasi-isomorphic to bounded complexes of free $k E$-modules. There is a localization sequence

$$
\mathbb{K}^{b}(\operatorname{proj}) \longrightarrow \mathrm{D}^{\mathrm{b}}(k E \text {-mod }) \longrightarrow k E \text {-stmod }
$$

(see [12]). Denote the corresponding localization functor by $\mathcal{R}$ :

$$
\mathcal{R}: \mathrm{D}^{\mathrm{b}}(k E \text {-mod }) \rightarrow k E \text {-stmod. }
$$

We define $\mathcal{G}: \mathrm{D}^{\mathrm{b}}(\mathcal{O}$-mod $) \rightarrow k E$-stmod as a composition $\mathcal{G}=\mathcal{R} \circ \widetilde{\mathcal{G}}$ :

$$
\mathcal{G}: \mathrm{D}^{\mathrm{b}}(\mathcal{O}-\text { mod }) \stackrel{\widetilde{\mathcal{G}}}{\longrightarrow} \mathrm{D}^{\mathrm{b}}(k E \text {-mod }) \stackrel{\mathcal{R}}{\longrightarrow} k E \text {-stmod } .
$$

Since $\mathcal{R}$ is a triangulated functor, Proposition 4.1 implies that $\mathcal{G}$ is a triangulated functor. 
The proof of the main theorem (Theorem 1.1) will rely on the following two properties of the functor $\mathcal{G}$ to be proved in Section 5 .

(1) Let $\mathcal{F}$ be an algebraic vector bundle of rank $s$ on $\mathbb{P}^{r-1}$. Consider $\mathcal{F}$ as a complex in $\mathrm{D}^{\mathrm{b}}(\mathcal{O}$-mod) concentrated in degree 0 . Then $\mathcal{G}(\mathcal{F})$ is a module of stable constant Jordan type $[1]^{s}$.

(2) Under the assumption of (1), we have

$$
\mathcal{F}_{1}(\mathcal{G}(\mathcal{F})) \simeq \begin{cases}\mathcal{F} & \text { if } p=2, \\ F^{*}(\mathcal{F}) & \text { if } p \text { is odd, }\end{cases}
$$

where $F: \mathbb{P}^{r-1} \rightarrow \mathbb{P}^{r-1}$ is the Frobenius map.

\section{Proof of the Main theorem}

We start with several technical lemmas. Because of the difference in structure of the cohomology ring, we divide the initial discussion into two cases, according to $p=2$ or $p$ odd.

Case $1(p=2)$. We define a $k$-algebra homomorphism

$$
\rho: H^{*}(E, k)=k\left[y_{1}, \ldots, y_{r}\right] \rightarrow k\left[Y_{1}, \ldots, Y_{r}\right]
$$

by $\rho\left(y_{i}\right)=Y_{i}$. Recall that we have an isomorphism $\mathcal{O}(-n)=\mathcal{F}_{1}(k)(-n) \simeq \mathcal{F}_{1}\left(\Omega^{n} k\right)$ by Remark 3.5.

Lemma 5.1. If $\zeta \in H^{n}(E, k)$ is represented by a cocycle $\hat{\zeta}: \Omega^{n+j} k \rightarrow \Omega^{j} k$ (with $j \in \mathbb{Z})$, then the diagram

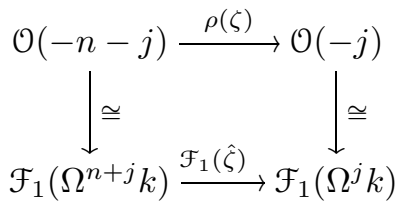

commutes.

Proof. Consider $\hat{\zeta}: \Omega^{n} k \rightarrow k$. The commutative diagram (3.2.2) applied to $\hat{\zeta}$ and iterated $j$ times becomes

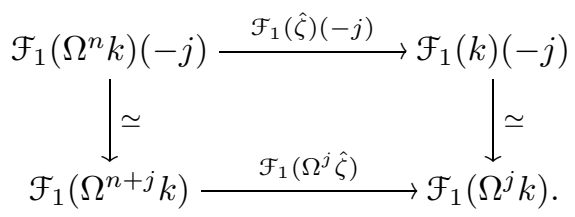

Hence, it suffices to assume that $j=0$. Additivity of the functor $\mathcal{F}_{1}$ allows us to assume that $\zeta$ is a monomial on generators $y_{1}, \ldots, y_{r}$. Finally, since multiplication in cohomology corresponds to composition of the corresponding maps on Heller shifts of $k$, it suffices to prove our statement for a degree one generator $\zeta=y_{i}$.

In the case $j=0, \zeta=y_{i}$, we need to show that the following diagram commutes (this is the diagram above twisted by $\mathcal{O}(1)$ ):

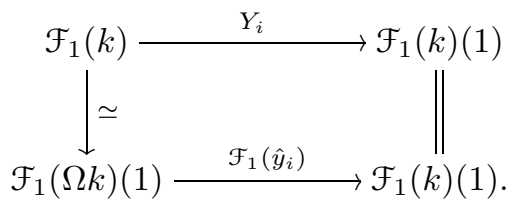


Let $E_{i}$ be the subgroup of index two in $E$ such that $y_{i}$ is inflated from $E / E_{i}$ to $E$, namely the subgroup generated by all of $g_{1}, \ldots, g_{r}$ except $g_{i}$. Then $y_{i}$ represents the class of the extension

$$
0 \rightarrow k \rightarrow M_{i} \rightarrow k \rightarrow 0
$$

where $M_{i}$ is the permutation module on the cosets of $E_{i}$. This is a length two module on which $X_{1}, \ldots, X_{r}$ act as zero except for $X_{i}$, which acts as a Jordan block of length two. We have a commutative diagram of $k E$-modules

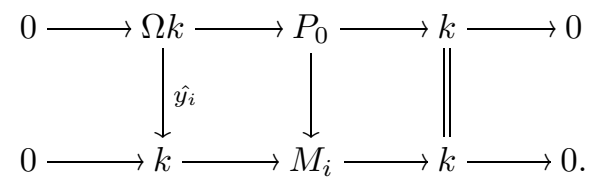

The left vertical isomorphism $\bar{\delta}: \mathcal{F}_{1}(k) \stackrel{\sim}{\rightarrow} \mathcal{F}_{1}(\Omega k)(1)$ of the diagram (5.1.1) is given by the switchback map for the short exact sequence

$$
0 \rightarrow \Omega k \rightarrow P_{0} \rightarrow k \rightarrow 0
$$

as in diagram (3.2.1). Applying $\theta$ to the commutative diagram on free $\mathcal{O}$-modules induced by the module diagram (5.1.2), we get a commutative diagram

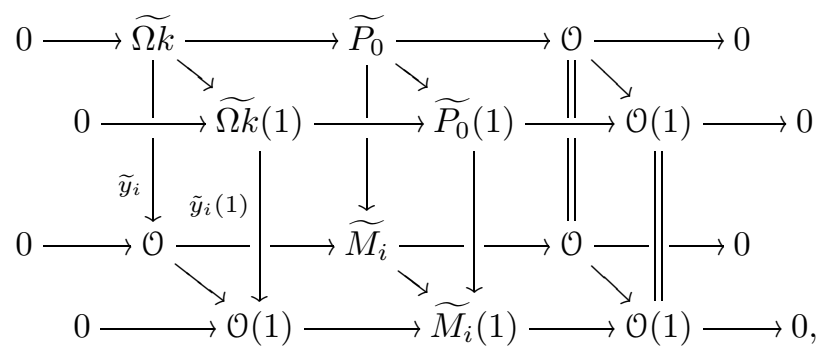

where all horizontal arrows going from back to front are given by the operator $\theta$ on the corresponding module. The map $\widetilde{y}_{i}: \widetilde{\Omega k} \rightarrow \mathcal{O}$ is induced by $\hat{y}_{i}: \Omega k \rightarrow k$. To compute the composite $\mathcal{F}_{1}\left(\hat{y}_{i}\right) \circ \bar{\delta}$ we first do the switchback map of the top layer and then push the result down via $\tilde{y}_{i}(1)$. Since the diagram is commutative, we can first push down via the identity map of the right vertical back arrow and then do the switchback of the bottom layer. Hence, the composite $\mathcal{F}_{1}\left(\hat{y}_{i}\right) \circ \bar{\delta}$ is given by the switchback map of the bottom layer, that is, of the diagram

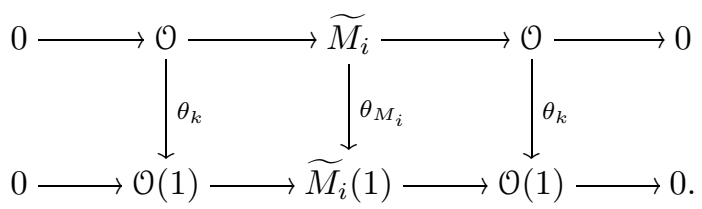

The left and right hand vertical maps here are zero. Hence, the switchback map $\bar{\delta}: \mathcal{O} \rightarrow \mathcal{O}(1)$ is given by multiplication by $\theta_{M_{i}}$, which is given by multiplication by $Y_{i}$ in this situation.

Case 2 ( $p$ is odd). We define a $k$-algebra homomorphism

$$
\rho: H^{*}(E, k) \rightarrow k\left[x_{1}, \ldots, x_{r}\right] \rightarrow k\left[Y_{1}, \ldots, Y_{r}\right]
$$

by $\rho\left(x_{i}\right)=Y_{i}^{p}$. 
Lemma 5.2. Let $p$ be an odd prime. Let $\zeta$ be a degree $n$ polynomial in $k\left[x_{1}, \ldots, x_{r}\right]$, regarded as an element of $H^{2 n}(E, k)$. If $\zeta$ is represented by a cocycle $\hat{\zeta}: \Omega^{2(n+j)} k \rightarrow$ $\Omega^{2 j} k$, then the diagram

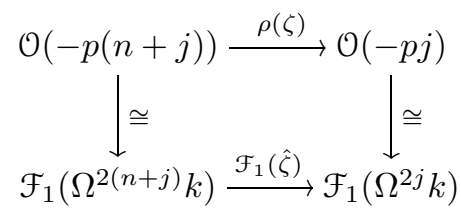

commutes.

Proof. The proof is similar to the proof in the case $p=2$, but more complicated. Again it suffices to treat the case where $\zeta=x_{i}$ and $j=0$. In other words, we need to compute the composite $\mathcal{F}_{1}\left(\hat{x}_{i}\right) \circ f$ in the diagram

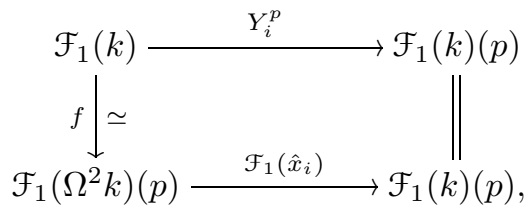

where $f: \mathcal{F}_{1}(k) \rightarrow \mathcal{F}_{1}\left(\Omega^{2} k\right)(p)$ is the isomorphism of Corollary 3.3. Let

$$
0 \longrightarrow \Omega^{2} k \longrightarrow P_{1} \longrightarrow P_{0} \longrightarrow k \longrightarrow 0
$$

be a truncated projective resolution of $k$. Tracing through the proof of Theorem 3.2 , we see that $f: \mathcal{F}_{1}(k) \rightarrow \mathcal{F}_{1}\left(\Omega^{2} k\right)(p)$ is a composite of three maps:

(1) the switchback of the top two rows of the diagram (5.2.1) below which gives the isomorphism $\mathcal{F}(k) \rightarrow \mathcal{F}_{p-1, p-2}(\Omega k)(1)$,

(2) followed by the isomorphism $\theta_{\Omega k}^{p-2}: \mathcal{F}_{p-1, p-2}(\Omega k)(1) \stackrel{\sim}{\rightarrow} \mathcal{F}_{p-1}(\Omega k)(p-1)$ of Lemma 2.3 ,

(3) followed by another switchback map, now for the bottom two rows of diagram (5.2.1), which gives the isomorphism $\mathcal{F}_{p-1}(\Omega k)(p-1) \simeq \mathcal{F}_{1}\left(\Omega^{2} k\right)(p)$ :

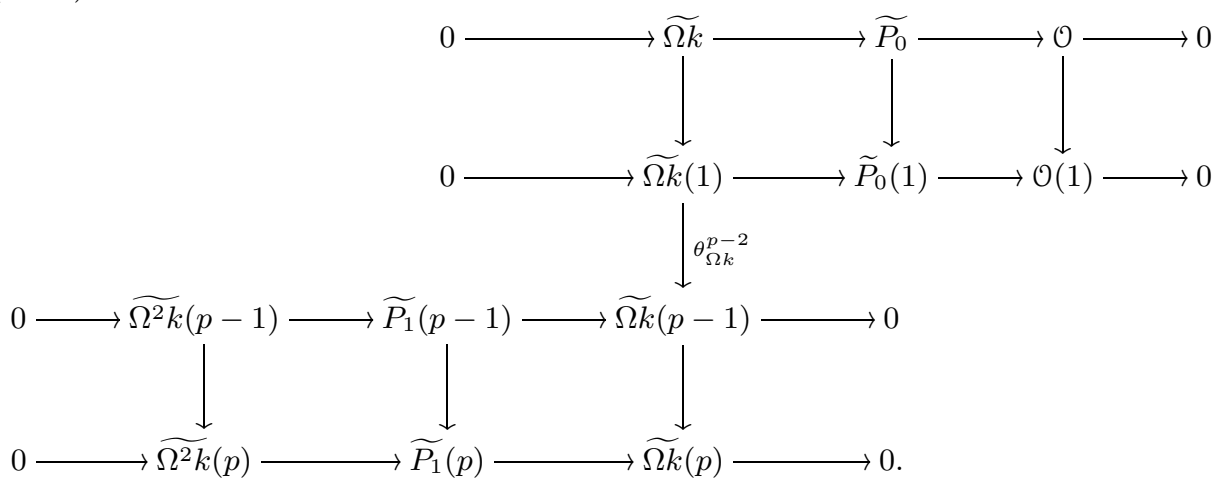

Let $E_{i}$ be the subgroup of index $p$ such that $x_{i}$ is inflated from $E / E_{i}$, namely the subgroup generated by all of $g_{1}, \ldots, g_{r}$ except for $g_{i}$. We let $M_{i}$ be the permutation module on the cosets of $E_{i}$. This is a length $p$ module on which $X_{1}, \ldots, X_{r}$ act as 
zero except for $X_{i}$, which acts as a Jordan block of length $p$. Then $x_{i}$ represents the class of the 2 -fold extension

$$
0 \longrightarrow k \longrightarrow M_{i} \longrightarrow M_{i} \longrightarrow k \longrightarrow 0,
$$

where the middle map is multiplication by $X_{i}$. We construct a diagram analogous to (5.2.1) for this extension:

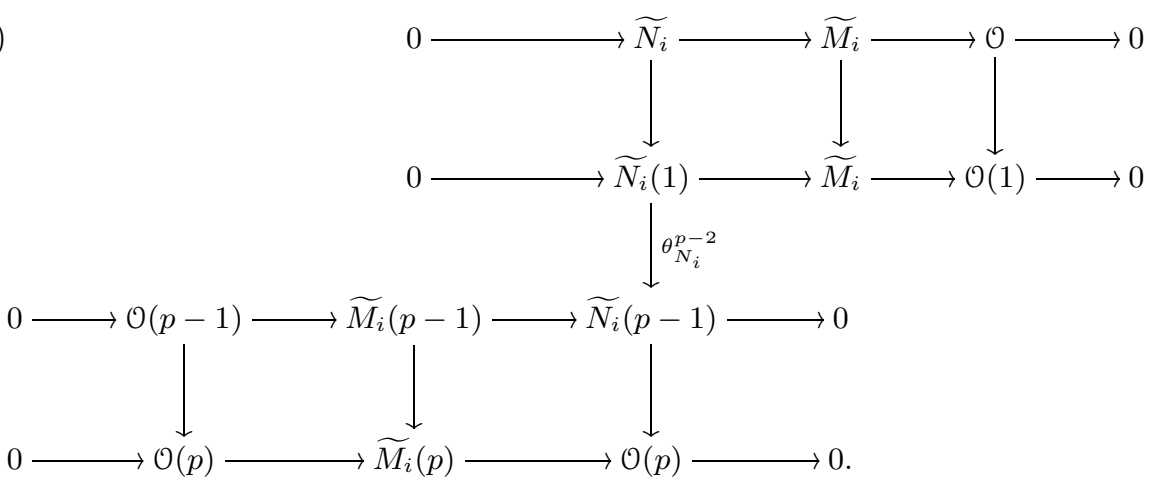

Here, $N_{i}=\operatorname{Im}\left\{X_{i}: M_{i} \rightarrow M_{i}\right\}$. Just as in the proof of Lemma 5.2, the module diagram

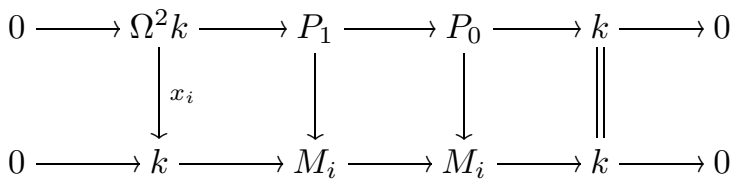

induces a commutative diagram of vector bundles with (5.2.1) on top and (5.2.2) at the bottom. Arguing as in the proof of Lemma 5.1, we compute the composite $\mathcal{F}_{1}\left(\hat{x}_{i}\right) \circ f$ by first mapping the rightmost $\mathcal{O}$ of diagram (5.2.1) identically to the rightmost $\mathcal{O}$ of diagram (5.2.2), and then applying our composite of a switchback, followed by $\theta_{N_{i}}^{p-2}$, followed by another switchback in the diagram (5.2.2). The maps $\theta_{M_{i}}: \widetilde{M}_{i} \rightarrow \widetilde{M}_{i}(1)$ and $\theta_{N_{i}}: \widetilde{N}_{i} \rightarrow \widetilde{N}_{i}(1)$ are simply multiplication by $Y_{i}$. Since the leftmost and rightmost vertical arrows in (5.2.2) are zero, to compute the composite of the three maps involved in diagram (5.2.2), we have to multiply first by $Y_{i}$, then by $Y_{i}^{p-2}$, then by $Y_{i}$ again. Hence, $\mathcal{F}_{1}\left(\hat{x}_{i}\right) \circ f=Y_{i}^{p}$.

Let

$$
F: \mathbb{P}^{r-1} \rightarrow \mathbb{P}^{r-1}
$$

be the Frobenius morphism, that is, the morphism given on the level of homogeneous coordinate algebras by

$$
k\left[Y_{1}, \ldots, Y_{r}\right] \stackrel{Y_{i} \mapsto Y_{i}^{p}}{\longrightarrow} k\left[Y_{1}, \ldots, Y_{r}\right] .
$$

Then the pullback functor

$$
F^{*}: \mathcal{O}-\bmod \rightarrow \mathcal{O}-\bmod
$$

is exact and induces a functor

$$
F^{*}: \mathrm{D}^{\mathrm{b}}(\mathcal{O}-\bmod ) \rightarrow \mathrm{D}^{\mathrm{b}}(\mathcal{O}-\bmod ) .
$$


In the next proposition we shall use the functor $\widetilde{\mathcal{G}}: \mathrm{D}^{\mathrm{b}}(\mathcal{O}-$ mod $) \rightarrow \mathrm{D}^{\mathrm{b}}(k E$-mod $)$ defined in Section 4

Proposition 5.3. Let

$$
\mathbf{C}=\bigoplus_{j=1}^{m_{n}} \mathcal{O}\left(a_{n, j}\right) \rightarrow \cdots \rightarrow \bigoplus_{j=1}^{m_{1}} \mathcal{O}\left(a_{1, j}\right) \rightarrow \bigoplus_{j=1}^{m_{0}} \mathcal{O}\left(a_{0, j}\right)
$$

be a complex of $\mathcal{O}-$ modules. Then

$$
\mathcal{F}_{1}(\widetilde{\mathcal{G}}(\mathbf{C}))= \begin{cases}\mathbf{C} & p=2 \\ F^{*}(\mathbf{C}) & p \text { odd } .\end{cases}
$$

Proof. By definition,

$$
\widetilde{\mathcal{G}}(\mathbf{C})=\bigoplus_{j=1}^{m_{n}} \Omega^{-\varepsilon a_{n, j}}(k) \rightarrow \cdots \rightarrow \bigoplus_{j=1}^{m_{1}} \Omega^{-\varepsilon a_{1, j}}(k) \rightarrow \bigoplus_{j=1}^{m_{0}} \Omega^{-\varepsilon a_{0, j}}(k) .
$$

Applying $\mathcal{F}_{1}$ term by term, we get

$$
\bigoplus_{j=1}^{m_{n}} \mathcal{F}_{1}\left(\Omega^{-\varepsilon a_{n, j}}(k)\right) \rightarrow \cdots \rightarrow \bigoplus_{j=1}^{m_{1}} \mathcal{F}_{1}\left(\Omega^{-\varepsilon a_{1, j}}(k)\right) \rightarrow \bigoplus_{j=1}^{m_{0}} \mathcal{F}_{1}\left(\Omega^{-\varepsilon a_{0, j}}(k)\right) .
$$

The statement now follows from Lemma 5.1 for $p=2$ and Lemma 5.2 for $p$ odd.

The following lemma will be applied to modules over $K\left[X_{\alpha}\right] /\left(X_{\alpha}^{p}\right)$, where projective and injective modules coincide.

Lemma 5.4. Let $\phi=\left(\begin{array}{ll}a & b \\ c & d\end{array}\right): A \oplus P \rightarrow B \oplus Q$ be an injective map of modules, with $a: A \rightarrow B$ a split injection, $P$ and $Q$ projective and injective. Then $\phi$ is a split injection.

Proof. Let $B=B_{1} \oplus B_{2}$, where $B_{1}=\operatorname{Im} a$, and let

$$
a=\left(\begin{array}{c}
a_{1} \\
0
\end{array}\right): A \rightarrow B_{1} \oplus B_{2}, \quad b=\left(\begin{array}{l}
b_{1} \\
b_{2}
\end{array}\right): P \rightarrow B_{1} \oplus B_{2} .
$$

With this notation, we have

$$
\phi=\left(\begin{array}{cc}
a_{1} & b_{1} \\
0 & b_{2} \\
c & d
\end{array}\right): A \oplus P \rightarrow B_{1} \oplus B_{2} \oplus Q .
$$

Since $a_{1}$ is an isomorphism, there exist maps $u: B_{1} \rightarrow Q, v: P \rightarrow A$ such that $c=u a_{1}$ and $b_{1}=a_{1} v$. Hence,

$$
\left(\begin{array}{ccc}
1 & 0 & 0 \\
0 & 1 & 0 \\
-u & 0 & 1
\end{array}\right)\left(\begin{array}{cc}
a_{1} & b_{1} \\
0 & b_{2} \\
c & d
\end{array}\right)\left(\begin{array}{cc}
1 & -v \\
0 & 1
\end{array}\right)=\left(\begin{array}{cc}
a_{1} & 0 \\
0 & b_{2} \\
0 & d-u b_{1}
\end{array}\right): A \oplus P \rightarrow B_{1} \oplus B_{2} \oplus Q
$$

By assumption, this map is injective. Hence, the map

$$
\left(\begin{array}{c}
b_{2} \\
d-u b_{1}
\end{array}\right): P \rightarrow B_{2} \oplus Q
$$

is injective and therefore split. 
Lemma 5.5. Let $0 \rightarrow A \rightarrow B \rightarrow C \rightarrow 0$ be a short exact sequence of $k E$-modules, such that $A$ and $B$ have trivial stable constant Jordan type and $\mathcal{F}_{1}(A) \rightarrow \mathcal{F}_{1}(B)$ is injective. Then $C$ is of trivial stable constant Jordan type and $\mathcal{F}_{1}(C)$ is the cokernel of $\mathcal{F}_{1}(A) \rightarrow \mathcal{F}_{1}(B)$.

Proof. Applying Lemma 5.4 to the restriction of the sequence to $K\left[X_{\alpha}\right] /\left(X_{\alpha}^{p}\right)$ for each $0 \neq \alpha \in \mathbb{A}^{r}(k)$, we conclude that this sequence is locally split. Hence, $C$ is a module of trivial constant Jordan type. By Proposition 3.7, applying $\mathcal{F}_{1}$ gives an exact sequence

$$
0 \rightarrow \mathcal{F}_{1}(A) \rightarrow \mathcal{F}_{1}(B) \rightarrow \mathcal{F}_{1}(C) \rightarrow 0 .
$$

We are now ready to prove the main theorem.

Theorem 5.6. Given any vector bundle $\mathcal{F}$ of rank $s$ on $\mathbb{P}^{r-1}$, there exists a finitely generated $k E$-module $M$ of stable constant Jordan type $[1]^{s}$ such that

(i) if $p=2$, then $\mathcal{F}_{1}(M) \cong \mathcal{F}$;

(ii) if $p$ is odd, then $\mathcal{F}_{1}(M) \cong F^{*}(\mathcal{F})$, the pullback of $\mathcal{F}$ along the Frobenius morphism $F: \mathbb{P}^{r-1} \rightarrow \mathbb{P}^{r-1}$.

Proof. Given a vector bundle $\mathcal{F}$ on $\mathbb{P}^{r-1}$, using the Hilbert syzygy theorem we can form a resolution by sums of twists of the structure sheaf:

$$
0 \rightarrow \bigoplus_{j=1}^{m_{r}} \mathcal{O}\left(a_{r, j}\right) \rightarrow \cdots \rightarrow \bigoplus_{j=1}^{m_{1}} \mathcal{O}\left(a_{1, j}\right) \rightarrow \bigoplus_{j=1}^{m_{0}} \mathcal{O}\left(a_{0, j}\right) \rightarrow \mathcal{F} \rightarrow 0 .
$$

Equivalently, $\mathcal{F}$, considered as a complex concentrated in degree 0 , is quasi-isomorphic to the complex

$$
\mathbf{C}=\bigoplus_{j=1}^{m_{r}} \mathcal{O}\left(a_{r, j}\right) \rightarrow \cdots \rightarrow \bigoplus_{j=1}^{m_{1}} \mathcal{O}\left(a_{1, j}\right) \rightarrow \bigoplus_{j=1}^{m_{0}} \mathcal{O}\left(a_{0, j}\right)
$$

Let

$$
M=\mathcal{G}(\mathcal{F}),
$$

where $\mathcal{G}: \mathrm{D}^{\mathrm{b}}(\mathcal{O}$-mod $) \rightarrow k E$-stmod is the functor defined towards the end of Section 4. We shall prove that $M$ satisfies the conclusions of the theorem.

Let

$$
\mathbf{C}_{\geq i}=\bigoplus_{j=1}^{m_{r}} \mathcal{O}\left(a_{r, j}\right) \rightarrow \cdots \rightarrow \bigoplus_{j=1}^{m_{i}} \mathcal{O}\left(a_{i, j}\right)
$$

be a truncation of $\mathbf{C}, 0 \leq i \leq r$. We set $M_{i}=\mathcal{G}\left(\mathbf{C}_{\geq i}\right)$. In particular, $M_{0}=M$. We shall show by the downward induction on $i$ that $M_{i}$ has trivial stable constant Jordan type and that $\mathcal{F}_{1}\left(M_{i}\right)$ is the cokernel of the rightmost map in the complex $\mathbf{C}_{\geq i}$ for $p=2$ or $F^{*}\left(\mathbf{C}_{\geq i}\right)$ for $p$ odd. Applied to $i=0$, this will give us the desired result about $M_{0}=M$.

By definition, $\mathcal{G}=\mathcal{R} \circ \widetilde{\mathcal{G}}$. Furthermore, we have

$$
\widetilde{\mathcal{G}}(\mathbf{C})=\bigoplus_{j=1}^{m_{r}} \Omega^{-\varepsilon a_{r, j}}(k) \rightarrow \cdots \rightarrow \bigoplus_{j=1}^{m_{1}} \Omega^{-\varepsilon a_{1, j}}(k) \rightarrow \bigoplus_{j=1}^{m_{0}} \Omega^{-\varepsilon a_{0, j}}(k) .
$$

Since $\mathcal{G}$ preserves triangles, the triangle

$$
\mathbf{C}_{\geq i+1} \rightarrow \mathbf{C}_{i} \rightarrow \mathbf{C}_{\geq i}
$$


in $\mathrm{D}^{\mathrm{b}}(\mathcal{O}$-mod $)$ gives rise to the triangle

$$
M_{i+1} \rightarrow \bigoplus_{j=1}^{m_{i}} \Omega^{-\varepsilon a_{i, j}}(k) \rightarrow M_{i}
$$

in $\operatorname{stmod}(k E)$ for any $i, 0 \leq i \leq r-1$.

By Proposition 5.3, applying $\mathcal{F}_{1}$ to the complex (5.6.2), we obtain either the original complex (5.6.1) if $p=2$ or its Frobenius twist if $p$ is odd.

Since $M_{r}=\mathcal{G}\left(\mathbf{C}_{r}\right)=\bigoplus \Omega^{-\varepsilon a_{r, j}}(k)$, we immediately get that $M_{r}$ is a module of trivial stable constant Jordan type. Moreover, $\mathcal{F}_{1}\left(M_{r}\right) \rightarrow \mathcal{F}_{1}\left(\bigoplus \Omega^{-\varepsilon a_{r-1, j}}(k)\right)$ is an injection since it is either the original leftmost map of the complex $\mathbf{C}(p=2)$ or of the complex $F^{*}(\mathbf{C})$ ( $p$ odd). Therefore, we may apply Lemma 5.5 to the triangle

$$
M_{r} \rightarrow \bigoplus_{j=1}^{m_{r-1}} \Omega^{-\varepsilon a_{r-1, j}}(k) \rightarrow M_{r-1} .
$$

This implies that $M_{r-1}$ has trivial stable constant Jordan type and that $\mathcal{F}_{1}\left(M_{r-1}\right)$ is the cokernel of the rightmost map of the complex $\mathbf{C}_{\geq(r-1)}(p=2)$ or the complex $F^{*}\left(\mathbf{C}_{\geq(r-1)}\right)$ ( $p$ odd). Proceeding in the same manner by downwards induction on $i$, we show that $M_{i}$ has trivial stable constant Jordan type and that $\mathcal{F}_{1}\left(M_{i}\right)$ is the cokernel of the rightmost map of the complex $\mathbf{C}_{\geq i}(p=2)$ or the complex $F^{*}\left(\mathbf{C}_{\geq i}\right)$ ( $p$ odd) for any $i, 0 \leq i \leq r-1$. Hence, for $i=0$ we get that $M=M_{0}$ has trivial stable constant Jordan type and that $\mathcal{F}_{1}(M)$ is the cokernel of $\mathbf{C}$, the resolution of $\mathcal{F}(p=2)$ or the cokernel of $F^{*}(\mathbf{C})$ ( $p$ odd) which is a resolution of $F^{*}(\mathcal{F})$ since $F^{*}$ is exact. Therefore, $\mathcal{F}_{1}(M) \simeq \mathcal{F}$ for $p=2$ and $\mathcal{F}_{1}(M) \simeq F^{*}(\mathcal{F})$ for $p$ odd. The fact that the stable type of $M$ is $[1]^{s}$ now follows from Proposition 2.1.

\section{Chern numbers}

Recall that the Chow ring of $\mathbb{P}^{r-1}$ is

$$
A^{*}\left(\mathbb{P}^{r-1}\right) \cong \mathbb{Z}[h] /\left(h^{r}\right) .
$$

If $\mathcal{F}$ is a vector bundle on $\mathbb{P}^{r-1}$, we write

$$
c(\mathcal{F}, h)=\sum_{j \geq 0} c_{j}(\mathcal{F}) h^{j} \in A^{*}\left(\mathbb{P}^{r-1}\right)
$$

for the Chern polynomial, where $c_{0}(\mathcal{F})=1$ and the $c_{i}(\mathcal{F}) \in \mathbb{Z}(1 \leq i \leq r-1)$ are the Chern numbers of $\mathcal{F}$.

If $0 \rightarrow \mathcal{F} \rightarrow \mathcal{F}^{\prime} \rightarrow \mathcal{F}^{\prime \prime} \rightarrow 0$ is a short exact sequence of vector bundles, then we have the Whitney sum formula

$$
c\left(\mathcal{F}^{\prime}, h\right)=c(\mathcal{F}, h) c\left(\mathcal{F}^{\prime \prime}, h\right) .
$$

Lemma 6.1. The formula for Chern numbers of twists of a rank $s$ vector bundle is

$$
c_{m}(\mathcal{F}(i))=\sum_{j=0}^{m} i^{j}\left(\begin{array}{c}
s-m+j \\
j
\end{array}\right) c_{m-j}(\mathcal{F}) .
$$

Equivalently, the total Chern class of the twists is given by

$$
c(\mathcal{F}(i), h)=\sum_{n=0}^{s} c_{n}(\mathcal{F}) h^{n}(1+i h)^{s-n} .
$$

Proof. See Fulton [9, Example 3.2.2]. 
More explicitly,

$$
\begin{aligned}
& c_{1}(\mathcal{F}(i))=c_{1}(\mathcal{F})+i s, \\
& c_{2}(\mathcal{F}(i))=c_{2}(\mathcal{F})+i(s-1) c_{1}(\mathcal{F})+i^{2}\left(\begin{array}{l}
s \\
2
\end{array}\right), \\
& c_{3}(\mathcal{F}(i))=c_{3}(\mathcal{F})+i(s-2) c_{2}(\mathcal{F})+i^{2}\left(\begin{array}{c}
s-1 \\
2
\end{array}\right) c_{1}(\mathcal{F})+i^{3}\left(\begin{array}{l}
s \\
3
\end{array}\right),
\end{aligned}
$$

and so on.

Lemma 6.2. For a vector bundle $\mathcal{F}$ of rank $s$ on $\mathbb{P}^{r-1}$ we have

$$
c(\mathcal{F}, h) c(\mathcal{F}(1), h) \cdots c(\mathcal{F}(p-1), h) \equiv 1-s h^{p-1} \quad\left(\bmod \left(p, h^{p}\right)\right) .
$$

Proof. We write

$$
c(\mathcal{F})=\prod_{j=1}^{s}\left(1+\alpha_{j} h\right),
$$

where the $\alpha_{j}$ are the Chern roots. Then the formula (6.1.1) is equivalent to

$$
c(\mathcal{F}(i))=\prod_{j=1}^{s}\left(1+\left(\alpha_{j}+i\right) h\right) .
$$

Thus we have

$$
c(\mathcal{F}) c(\mathcal{F}(1)) \cdots c(\mathcal{F}(p-1))=\prod_{j=1}^{s}\left(1+\alpha_{j} h\right)\left(1+\left(\alpha_{j}+1\right) h\right) \cdots\left(1+\left(\alpha_{j}+p-1\right) h\right) .
$$

Now by Fermat's little theorem, we have the identity

$$
x(x+y) \cdots(x+(p-1) y) \equiv x^{p}-x y^{p-1} \quad(\bmod p)
$$

and so putting $x=1+\alpha_{j} h, y=h$ we obtain

$$
\begin{aligned}
c(\mathcal{F}) c(\mathcal{F}(1)) \cdots c(\mathcal{F}(p-1)) & \equiv \prod_{j=1}^{s}\left(\left(1+\alpha_{j} h\right)^{p}-\left(1+\alpha_{j} h\right) h^{p-1}\right) \quad(\bmod p) \\
& \equiv \prod_{j=1}^{s}\left(1-h^{p-1}+\left(\alpha_{j}^{p}-\alpha_{j}\right) h^{p}\right) \quad(\bmod p) \\
& \equiv 1-s h^{p-1} \quad\left(\bmod \left(p, h^{p}\right)\right) .
\end{aligned}
$$

A priori, this is a congruence between polynomials with algebraic integer coefficients. But if two rational integers are congruent $\bmod p$ as algebraic integers, then they are also congruent modulo $p$ as rational integers. This is because their difference, divided by $p$, is both an algebraic integer and a rational number, therefore an integer.

We now restate and prove Theorem 1.2 .

Theorem 6.3. Suppose that $M$ has stable constant Jordan type $[1]^{s}$. Then $p$ divides the Chern numbers $c_{m}\left(\mathcal{F}_{1}(M)\right)$ for $1 \leq m \leq p-2$.

Proof. Since $M$ has stable Jordan type $[1]^{s}$, we have $\mathcal{F}_{2}(M)=\ldots=\mathcal{F}_{p-1}(M)=0$. Hence, the trivial vector bundle $\widetilde{M}$ has a filtration with filtered quotients (not in 
order) $\mathcal{F}_{1}(M), \mathcal{F}_{p}(M), \mathcal{F}_{p}(M)(1), \ldots, \mathcal{F}_{p}(M)(p-1)$. So we have

$$
\begin{aligned}
1 & =c(\widetilde{M}, h) \\
& =c\left(\mathcal{F}_{1}(M), h\right) c\left(\mathcal{F}_{p}(M), h\right) c\left(\mathcal{F}_{p}(M)(1), h\right) \cdots c\left(\mathcal{F}_{p}(M)(p-1), h\right) \\
& \equiv c\left(\mathcal{F}_{1}(M), h\right) \quad\left(\bmod \left(p, h^{p-1}\right)\right)
\end{aligned}
$$

by Lemma 6.2. It follows that the coefficients $c_{m}\left(\mathcal{F}_{1}(M)\right)$ are divisible by $p$ for $1 \leq m \leq p-2$.

Remark 6.4. For $p=2$ this theorem says nothing. But for $p$ odd, it at least forces $c_{1}\left(\mathcal{F}_{1}(M)\right)$ to be divisible by $p$. As an explicit example, the twists of the HorrocksMumford bundle $\mathcal{F}_{\mathrm{HM}}(i)$ have $c_{1}=2 i+5$ and $c_{2}=i^{2}+5 i+10$ (11). For $p \geq 7$ these cannot both be divisible by $p$, and so there is no module $M$ of stable constant Jordan type $[1]^{2}$ and integer $i$ such that $\mathcal{F}_{1}(M) \cong \mathcal{F}_{\text {HM }}(i)$.

Remark 6.5. The conclusion of the theorem is limited to the modules of stable constant Jordan type [1] $]^{s}$. For example, if $M_{n}$ is a "zig-zag" module of dimension $2 n+1$ for $\mathbb{Z} / p \times \mathbb{Z} / p$, then $\mathcal{F}_{1}\left(M_{n}\right) \simeq \mathcal{O}(-n)$ and $\mathcal{F}_{1}\left(M_{n}^{*}\right) \simeq \mathcal{O}(n)$ for any $n \geq 0$ (see [7, §6]).

\section{ACKNOWLEDGEMENT}

Both authors are grateful to MSRI for its hospitality during the 2008 program "Representation theory of finite groups and related topics" where much of this research was carried out. They also thank the referee for helpful suggestions.

\section{REFERENCES}

1. D. J. Benson, Modules of constant Jordan type and the Horrocks-Mumford bundle, preprint, 2008. MR 2376286 (2008j:20135)

2. _ Modules of constant Jordan type with one non-projective block, Algebras and Representation Theory 13 (2010), 315-318. MR2630123 (2011d:20015)

3. I. N. Bernšteĭn, I. M. Gel'fand, and S. I. Gel'fand, Algebraic vector bundles on $\mathbb{P}^{n}$ and problems of linear algebra, Funktsional. Anal. i Prilozhen. 12 (1978), no. 3, 66-67. MR509387 (80c:14010a)

4. J. F. Carlson and E. M. Friedlander, Exact category of modules of constant Jordan type, Algebra, arithmetic and geometry: Manin Festschrift, Progr. in Math., vol. 269, Birkhäuser Verlag, Basel, 2009, pp. 267-290. MR2641174(2011c:16035)

5. J. F. Carlson, E. M. Friedlander, and J. Pevtsova, Modules of constant Jordan type, J. Reine \& Angew. Math. 614 (2008), 191-234. MR2376286 (2008j:20135)

6. J. F. Carlson, E. M. Friedlander, and A. A. Suslin, Modules for $\mathbb{Z} / p \times \mathbb{Z} / p$, Comment. Math. Helv. 86 (2011), 609-657. MR2803855

7. E. M. Friedlander and J. Pevtsova, Constructions for infinitesimal group schemes, Trans. Amer. Math. Soc. 363 (2011), 6007-6061. MR2817418 (2012d:14081)

8. _ Generalized support varieties for finite group schemes, Documenta Math. Extra Volume Suslin (2010), 197-222. MR2804254

9. W. Fulton, Intersection theory, Ergebnisse der Mathematik und ihrer Grenzgebiete, Folge 3, Band 2, Springer-Verlag, Berlin/New York, 1984. MR732620 (85k:14004)

10. R. Hartshorne, Algebraic geometry, Graduate Texts in Mathematics, vol. 52, Springer-Verlag, Berlin/New York, 1977. MR0463157 (57:3116)

11. G. Horrocks and D. Mumford, A rank 2 vector bundle on $\mathbb{P}^{4}$ with 15,000 symmetries, Topology 12 (1973), 63-81. MR0382279 (52:3164) 
12. J. Rickard, Derived categories and stable equivalence, J. Pure \& Applied Algebra 61 (1989), 303-317. MR1027750 (91a:16004)

Department of Mathematics, University of Aberdeen, King's College, Meston Building, Aberdeen AB24 3UE, Scotland

Department of Mathematics, University of Washington, Seattle, Washington 98195 